\title{
FAMILY PRESENCE AND THE PRESS EFFECTS ON PREHOSPITAL CARDIOPULMONARY RESUSCITATION: ATTITUDES OF A GROUP OF EMERGENCY CAREGIVERS
}

\author{
HASAN ERBAY*
}

\begin{abstract}
There have been many studies regarding family presence during cardiopulmonary resuscitation and most of them are about emergency services or intensive care units. However, the issue has not been studied enough in terms of prehospital emergency medicine and the perspective of prehospital emergency caregivers. In this study, it is aimed to present the attitudes of a group of prehospital emergency care professionals to family presence and the press effects during prehospital cardiopulmonary resuscitation. The data for this descriptive research was collected from the participants of 63 prehospital caregivers in Afyonkarahisar. The data was collected using a questionnaire designed by the authors and the responses summarized by using frequencies and percentages. Descriptive statistics were used to characterize the sample and each of the survey items. The local ethics committee approval was obtained. Most participants were strongly opposed to family presence and the press during $C P R$. The highest mean in the phrases that "I don't want any press member to be there when I perform CPR on a patient." There is no significant difference in family presence during CPR between the role and year of the experience. Prehospital emergency caregivers mostly have negative attitude towards family presence and the press during CPR. They mostly think the presence of significant others and being watched adversely affects their CPR performance. Disturbing effect on caregivers is not only related to the presence of family members or to other significant others but also the press. Family presence and the press effect on prehospital cardiopulmonary resuscitation are crucial issues that need more attention.
\end{abstract}

Keywords: Prehospital emergency medicine; Ethics; Paramedics; The press; Healthcare for specific diseases/group.

\section{INTRODUCTION}

Cardiopulmonary resuscitation (CPR) includes the performance of chest compressions, airway management, rescue breathing, rhythm detection, and shock delivery (if indicated) by an integrated team of highly trained rescuers who are competent both for in-hospital and out-ofhospital settings to the casualties thought to be in cardiac arrest (American Heart Association 2015). Prehospital emergency caregivers (PECs) are confronted with a number of ethical considerations when they are on their way to treat a person who suffers an out-of-hospital cardiac arrest with CPR (Ågård et al. 2012; Brenner et al. 2018). One of the conflicts in the prehospital setting is family-witnessed CPR called family presence during CPR (FPDR) (Abelsson and Lindwall 2018; Erbay 2014; Karlsson, Karlsson, and Hilli 2019; Torabi et al. 2019).

It is a long-running debate and family presence during resuscitation means that the family members can witness the visual and/or physical contact of the caregivers with the patient during resuscitation (Leske, McAndrew, and Brasel 2013). There are several aspects of FPDR 
that concern the patient, their relatives, and emergency care providers (Bray et al. 2016; Jabre et al. 2013). In many studies, this issue has been evaluated from the perspective of the family members, and it has been shown that family members mostly have positive views on FPDR (Albarran et al. 2009; Masa'Deh et al. 2014; Parial, Torres, and Macindo 2016). Many specific studies also have investigated the perceptions of health care professionals toward FPDR, and they have presented various involvements of it revealing that the issue is not so clear from their side, and it contains many discussions (Moreland 2005; Parial et al. 2016). Although many associations including The Emergency Nurses Association (ENA), European Federation of Critical Nursing Associations (EfCCNa), European Society of Cardiology (ESC), Association of Cardiovascular Nursing \& Allied Professions (ACNAP), American Heart Association (AHA) and European Resuscitation Council (ERC) advocate for allowing the option of FPDR and recommend in-hospital family-witnessed resuscitation; for the most part, no guidance exists around how to support family members best especially in the prehospital settings (Brown 2016; Lippert et al. 2010; Sak-Dankosky et al. 2015).

However, FPDR is a controversial issue because of its ethical, cultural, and legal aspects and its effect of psychological or emotional trauma on the patient's relatives (Barreto et al. 2019; Dewitt 2015; Kirchhoff et al. 2007; Köberich et al. 2010; McClement, Fallis, and Pereira 2009; McLaughlin, Melby, and Coates 2013; Mian et al. 2007; De Robertis et al. 2017; Strasen, Van Sell, and Sheriff 2016; Yanturali et al. 2005). Moreover, discussions about FPDR are almost for in-hospital CPR (in the emergency room and intensive care units)(Goldberger et al. 2015); not about prehospital CPR. Most previous studies focus on the perceptions of physicians and nurses in hospital settings, but limited findings reported about the opinions of other CPR team members, including paramedics, emergency nurses, and emergency technicians (Walker 2014). PECs are at the forefront of providing immediate CPR to the patient usually in patient's home, and yet little is known on their experiences and perceptions of FPDR; whereas, family witnessing is sometimes inevitable in prehospital practice.

Besides, the other conflict on FPDR is the press, and press-related issues. Professionally, PECs prioritize CPR of the patient however; the thing that the press members prioritize is not CPR, but the "news" of this situation. Many issues may be counted as stress factors during CPR (Lundsgaard and Lundsgaard 2018); on the other hand, the presence of the press at the scene in prehospital settings may also be considered as one of these factors. The press effect in this regard has been reported scarcely in the literature.

This study aims to describe the attitudes of a group of prehospital emergency care professionals regarding the family presence and the press effect during prehospital cardiopulmonary resuscitation in Turkey. The term "family" in this paper, not only refers to close family members of the patient but also the significant others including friends, neighbors, or colleagues at the scene of CPR attempt.

\section{METHODS}

This was a descriptive study carried out between March-May 2015. The questionnaire was specifically designed for this research by the authors after review of the literature. Before starting the study, a preliminary questionnaire was conducted on 12 participants, and some of the statements and phrases in the form were modified. The 18 expressions in total from the data collection forms were re-evaluated by considering similar characteristics and rearranged in 10 items. Then, the study took place in Afyonkarahisar, Turkey. 


\section{Data Collection}

Data was collected using a structured questionnaire. It consisted of two sections: (a) Demographics (gender, role, and year of experience); (b) the section of 10 items each was scored on an 11-Point-Likert-Scale. Each participant was asked to evaluate the content of the questionnaire by evaluating each item on an eleven-point scale: $0=$ disagree, $5=$ no opinion/not sure and $10=$ agree.

\section{Data Analysis}

The data were analyzed using the Statistical Package for Social Scientists (SPSS, version 11.0 for windows: SPSS Inc., Chicago, IL, USA). Statistical advice was sought from a statistician to analyze the data. Descriptive statistics were used to characterize the sample and each of the survey items. Because of small sample and distribution of data, Mann-Whitney U test was used to compare differences between the gender, and Kruskal-Wallis H test was used to check the differences between the role and year of experience that the samples are independent.

\section{Ethical Considerations}

The approval of the study was obtained from the local Ethics Committee. The participants were informed of the purpose of the study, were assured of their right to refuse to participate or to withdraw at any stage, and that data would be anonymous.

\section{RESULTS}

Eighty-four PECs on active duty on their shift were enrolled in this study. The total number of the PECs in duty at the region was 129 at that time. The term PECs includes emergency physicians, emergency nurses, paramedics, emergency medical technicians and ambulance drivers. Eight declined to participate, seven forms were filled incomplete (at least three items incomplete), and six forms were excluded because of the answers were totally blank. Overall, final evaluations were made on the forms of sixty-three participants at hand. Among the 63 responders, for demographic findings of each group by highest rates; $38(60.32 \%)$ are male, 31 (49.21\%) are paramedics, and 37 (58.73\%) are 1-3 years work experience. The demographic findings of the PECs are shown in Figure 1.

Figure 1. Demographic Characteristics of the Participants.
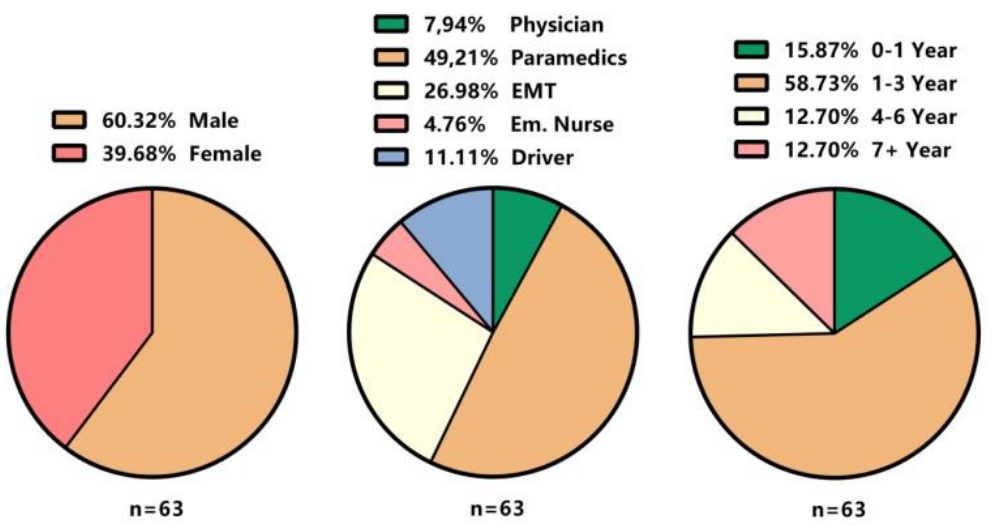
Responses of participants for ten phrases and distribution of the response with score and percentage are given in Table 1. Among the 63 responders, it is given the number of the participants, mean and standard deviation (SD) of the response. It is the highest mean in the sixth phrase that "I don't want any press member to be there when I perform CPR on a patient" (Figure 2). The lowest mean is in the seventh phrase that "The presence of press members when performing CPR to a patient positively affects my CPR performance" (Figure 3 ). The highest agree score is in the sixth phrase, and the lowest disagree score is in the fourth phrase that "When applying CPR to a patient, relatives can be present and witness our intervention if they request". Participants are not sure about the phrase that "I understand some people's desire to be with their loved ones at this difficult moment". The main theme of the study which is shown in the second phrase that "Having someone watching me while applying CPR to a patient, positively affects my performance on CPR" has the third-lowest mean.

Table 1. Participants' attitude toward family presence and the press during prehospital CPR and distribution of the response with score and percentage.

\begin{tabular}{|c|c|c|c|c|}
\hline Phrases & $\begin{array}{l}\text { Mean } \pm S D \\
n=63\end{array}$ & $\begin{array}{l}\text { Disagree } \\
\text { (points 0-3) }\end{array}$ & $\begin{array}{l}\text { Not sure } \\
\text { (points 4-6) }\end{array}$ & $\begin{array}{c}\text { Agree } \\
\text { (points 7-10) }\end{array}$ \\
\hline $\begin{array}{l}\text { 1. I don't want to do CPR while people } \\
\text { watching me. }\end{array}$ & $5,68 \pm 3,75$ & $\begin{array}{c}20 \\
(31.74 \%) \\
\end{array}$ & $\begin{array}{c}14 \\
(22.22 \%) \\
\end{array}$ & $\begin{array}{c}29 \\
(46.03 \%) \\
\end{array}$ \\
\hline $\begin{array}{l}\text { 2. Having someone watching me while } \\
\text { applying CPR to a patient, positively } \\
\text { affects my performance on CPR. }\end{array}$ & $2,89 \pm 2,82$ & $\begin{array}{c}37 \\
(58.73 \%)\end{array}$ & $\begin{array}{c}19 \\
(30.15 \%) \\
\end{array}$ & $\begin{array}{c}7 \\
(11.11 \%)\end{array}$ \\
\hline $\begin{array}{l}\text { 3. I think, having someone around watching } \\
\text { them while an ambulance crew is } \\
\text { performing CPR to the patient, negatively } \\
\text { affects the performance of the team. }\end{array}$ & $7,03 \pm 3,21$ & $\begin{array}{c}10 \\
(15.87 \%)\end{array}$ & $\begin{array}{c}13 \\
(20.63 \%)\end{array}$ & $\begin{array}{c}40 \\
(63.49 \%)\end{array}$ \\
\hline $\begin{array}{l}\text { 4. When applying CPR to a patient, relatives } \\
\text { can be present and witness our intervention } \\
\text { if they request. }\end{array}$ & $2,38 \pm 3,37$ & $\begin{array}{c}48 \\
(76.19 \%)\end{array}$ & $\begin{array}{c}5 \\
(7.93 \%)\end{array}$ & $\begin{array}{c}10 \\
(15.87 \%)\end{array}$ \\
\hline $\begin{array}{l}\text { 5. I think I would like to be there if I have the } \\
\text { opportunity when being applied CPR to } \\
\text { someone close to me. }\end{array}$ & $5,57 \pm 4,14$ & $\begin{array}{c}22 \\
(34.92 \%)\end{array}$ & $\begin{array}{c}12 \\
(19.04 \%)\end{array}$ & $\begin{array}{c}29 \\
(46.03 \%)\end{array}$ \\
\hline $\begin{array}{l}\text { 6. I don't want any press member to be there } \\
\text { when I perform CPR on a patient. }\end{array}$ & $8,49 \pm 3,01$ & $\begin{array}{c}6 \\
(9.52 \%) \\
\end{array}$ & $\begin{array}{c}6 \\
(9.52 \%) \\
\end{array}$ & $\begin{array}{c}51 \\
(80.95 \%) \\
\end{array}$ \\
\hline $\begin{array}{l}\text { 7. The presence of press members when } \\
\text { performing CPR to a patient positively } \\
\text { affects my CPR performance. }\end{array}$ & $1,97 \pm 2,78$ & $\begin{array}{c}47 \\
(74.60 \%)\end{array}$ & $\begin{array}{c}9 \\
(14.28 \%)\end{array}$ & $\begin{array}{c}7 \\
(11.11 \%)\end{array}$ \\
\hline $\begin{array}{l}\text { 8. I think that taking image or shooting video } \\
\text { by the press simultaneously when } \\
\text { performing CPR on a patient affects the } \\
\text { performance of the ambulance crew } \\
\text { negatively. }\end{array}$ & $6,84 \pm 3,71$ & $\begin{array}{c}15 \\
(23.80 \%)\end{array}$ & $\begin{array}{c}7 \\
(11.11 \%)\end{array}$ & $\begin{array}{c}41 \\
(65.07 \%)\end{array}$ \\
\hline $\begin{array}{l}\text { 9. I understand some people's desire to be } \\
\text { with their loved ones at this difficult } \\
\text { moment. }\end{array}$ & $5,33 \pm 3,41$ & $\begin{array}{c}20 \\
(31.74 \%)\end{array}$ & $\begin{array}{c}19 \\
(30.15 \%)\end{array}$ & $\begin{array}{c}24 \\
(38.09 \%)\end{array}$ \\
\hline $\begin{array}{l}\text { 10. I think the main reason that a person wants } \\
\text { to be there when CPR is applied to his } \\
\text { relative is that he wants to see that } \\
\text { everything should be done is really being } \\
\text { done. }\end{array}$ & $6,19 \pm 3,08$ & $\begin{array}{c}10 \\
(15.87 \%)\end{array}$ & $\begin{array}{c}18 \\
(28.57 \%)\end{array}$ & $\begin{array}{c}35 \\
(55.55 \%)\end{array}$ \\
\hline
\end{tabular}


Figure 2. Distribution of score for the phrase; "I don't want any press member to be there when I perform CPR on a patient." Values are expressed as Means $\pm \mathrm{SD}, \mathrm{n}=63$.

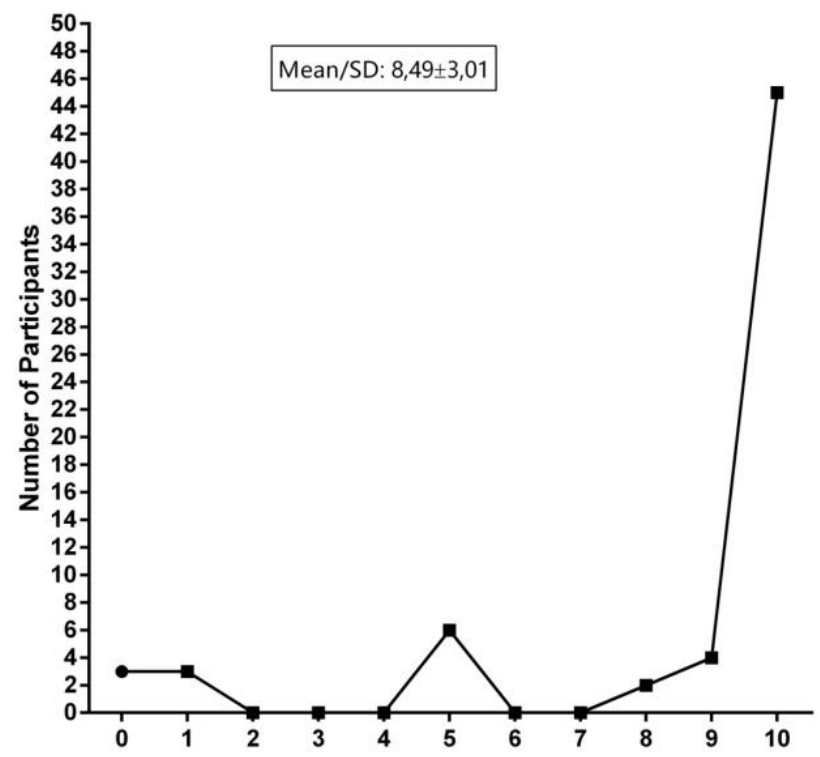

Figure 3. Distribution of score for the phrase; "The presence of press members when performing CPR to a patient positively affects my CPR performance." Values are expressed as Means $\pm \mathrm{SD}, \mathrm{n}=63$.

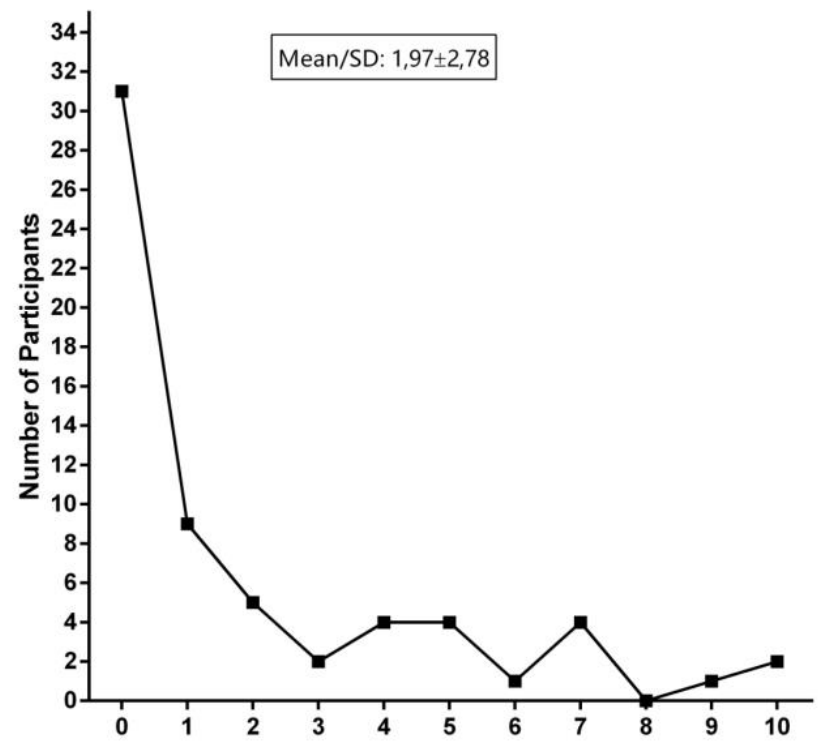

Female participants adopt the fifth phrase that "I think I would like to be there if I have the opportunity when being applied CPR to someone close to me" more than men (Figure 4). Finally, female participants adopt the tenth phrase that "I think the main reason that a person wants to be there when CPR is applied to his relative is that he wants to see that everything should be done is really being done" less than men (Figure 5). There is no significance in FPDR between the role and year of the experience in PECs. 
Figure 4. Gender difference for the phrase; "I think I would like to be there if I have the opportunity when being applied CPR to someone close to me." $n=38$ Male and 25 female participants. *significant at $\mathrm{P}<0.05$ as compared with Male.

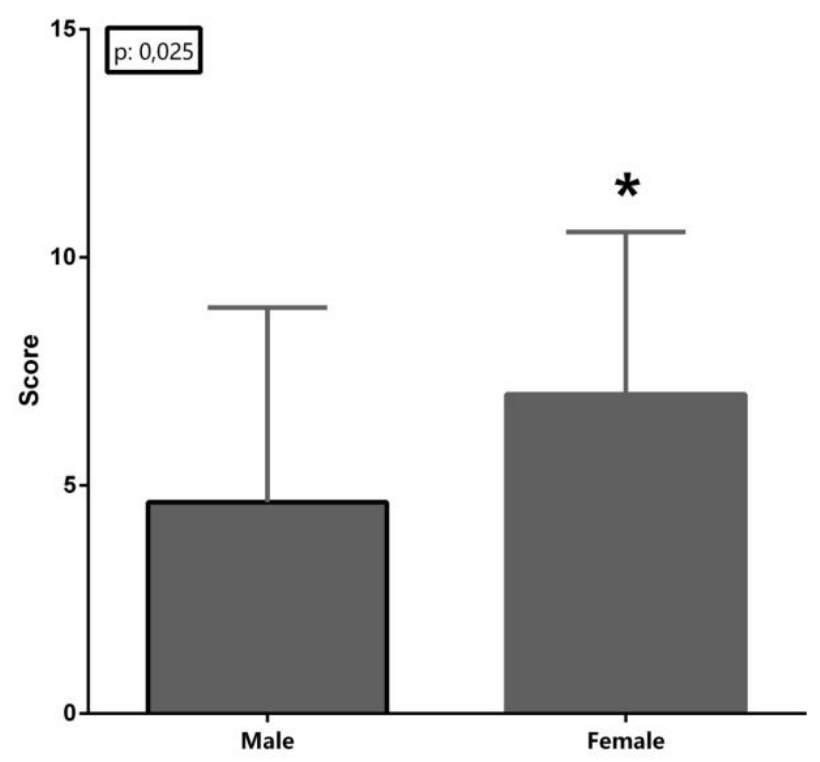

Figure 5. Gender difference for the phrase; "I think the main reason that a person wants to be there when CPR is applied to his relative is that he wants to see that everything should be done is really being done." $\mathrm{n}=38 \mathrm{Male}$ and 25 female participants. *significant at $\mathrm{P}<0.05$ as compared with Male.

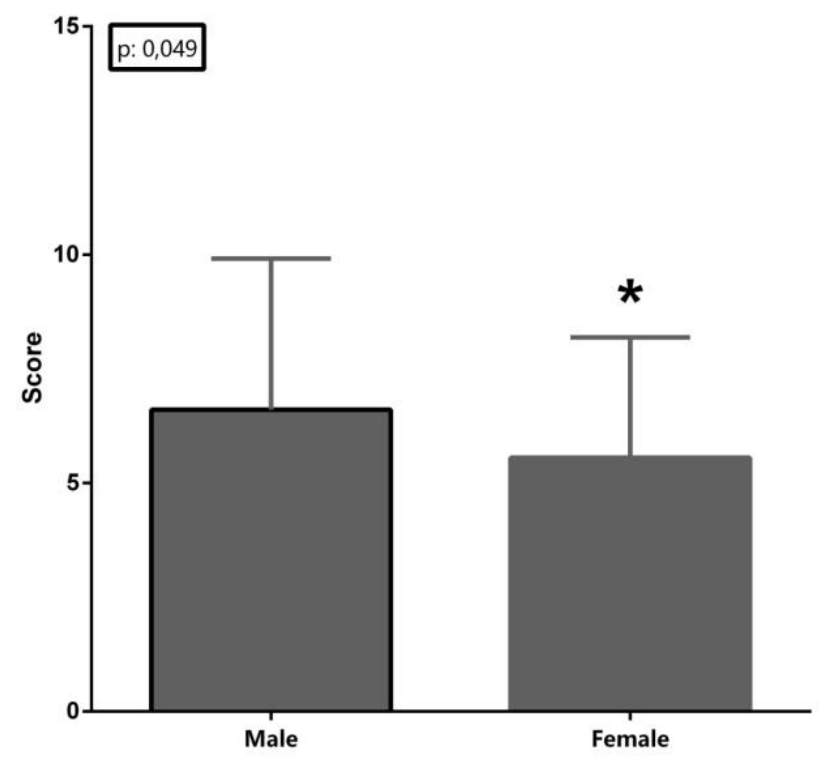

DISCUSSION

FPDR is fast-paced, physical, intense, and often emotional for both health care professionals and bystanders (Tennyson 2019). There is great variability in FPDR implementation in clinical practice. The issue of allowing the presence of patients' families by the health care providers is a paradigm shift (Barreto et al. 2019; ENA Clinical Practice Guideline Committee 2012). Some health care professionals have positive attitudes toward FPDR (Bae, Lee, and Jang 2008; 
Köberich et al. 2010; Lederman and Wacht 2014; MacLean et al. 2003; Meyers et al. 2000; Zavotsky et al. 2014), although some have concerns (Demir 2008; Pankop et al. 2013; Ramos et al. 2016; De Stefano et al. 2016). It is a pendulum oscillating between "benefits" and "disadvantages and/or limitations" (Barreto et al. 2019).

In this study, PECs mostly tend to have a negative attitude to the FPDR. It seems the most important reason for this could be that the justification of "being watched" which adversely affects CPR performance of the team. The presence of the patient's relatives watching PECs at the time could be reasonably the main factor in PECs' attitude. For some practices based on professional skills, such as CPR, it is important for practitioners to be free from outside factors affecting team performance. It is seen by the study findings that health professionals consider the feeling of "being watched" to be a negative external effect for their practice. However, according to some studies, PECs think they do not experience more stress when relatives are present because relatives do not interfere with the process (Jabre et al. 2013; Al Mutair et al. 2014; Walker 2014). This approach is probably due to two reasons; firstly PECs' perceptions that the patient's relatives do not intervene in the process, and secondly it is just experience. Therefore, the difference between two attitudes may be due to cultural and legal aspects that are changing country by country.

From the perspective of ethical conflicts experienced by PECs, it can be said that the conflict occurs between three broad principles; beneficence, nonmaleficence, and autonomy on FPDR. Giving relatives the autonomy to choose their involvement in such a critical moment is sure meaningful; however, there is an ethical conflict when deciding which one is more important; family's desire, patient's beneficence and/or maleficence. Although the basic principles of medical ethics appear to be accepted across cultures, the priority of these principles may vary among different cultures and ethics (American Heart Association 2000). The principle here that PECs give priority is beneficence. The study states that PECs think their CPR performance is adversely affected in the situation of "being watched". Hence, it means that FPDR is not something beneficence for the patient. So, FPDR is not only the subject of medicine but also sociology, culture, and of course, religion. This is probably the reason why many countries and societies have different findings on the issue. Of course, the perspective on end-of-life, death, and death-related issues are greatly influenced by cultural elements (Blank 2011). In that respect, the results of the present study are the same with some studies results on the negative attitude of health care providers towards FPDR in Turkey (Badir and Sepit 2007; Demir 2008; Güneş and Zaybak 2009; Yanturali et al. 2005).

This study reveals that PECs are not only familiar with the negative effect of the concept of FPDR on their own team performance in particular, but also the negative effects of other PECs team CPR performance. CPR performance is essential for linking the patient to the chain of survival for treating cardiac arrest. However, the performance is highly variable both in outof-hospital and in-hospital CPR (Hostler et al. 2011; Wik et al. 2005). These performance concerns in PECs' minds may also create extra stress on them. Eventually, this negative approach may be due to increased stress burden in PECs during CPR and the atmosphere of the event. This anxiety also applies to possible violence and abuse (Köberich et al. 2010). PECs remain anxious over possible threats of violence and abuse from significant distressed others (Torabi et al. 2019).

PECs generally perform CPR at the patient's home, and effective communication with patient's relatives is important during this process (De Stefano et al. 2016). FPDR, especially in elderly patients, can have positive contributions in terms of accepting the impending death of the patient, communication, and making a possible contribution to health professionals (Tennyson 2019). However, it is very difficult to achieve a good verbal communication with the patient and bystanders, if only one PECs team arrives at the home (Karlsson et al. 2019). Because there is too much team-work to do systematically, repeatedly, and successfully for 
CPR attempt in a short time, but often there is only one team. This situation is a challenge to manage the procedure easily and is one of the unique structures of the prehospital settings that include many conflicts (Erbay 2014). Some systematic reviews report lower survival rates after cardiac resuscitation (Berdowski et al. 2010; Sasson et al. 2010). Thus, in such a case that mortality is so high, and immediate action is needed within the limited time, the request of PECs to be on their own in this process, is understandable. PECs mostly face to make a crucial decision, and they surely need to focus on what they are doing. In such a critical situation, effective communication may obviously not be among the priorities of PECs.

During a medical process that highly concerns one's life, it is an ethical necessity to minimize all the factors that may have negative impacts on the performance of health care professionals. The discussions on the FPDR need to be addressed in this respect related to reduce negative impacts and help health care providers. In this study, it may be the main reason for being there when applying CPR to someone close them; in the case they consider the possibility to help the CPR team there as if they are the health professionals, too. Of course, it is highly emotionally challenging position and at that point, as a bystander, they tend to accept their position not as an ordinary relative but as a health professional who knows the technical details of CPR. Thus, it may increase empathy with PECs and improve CPR efforts.

One of the prominent findings of the present study is that PECs are strongly opposed especially to the presence of the press members during CPR. This opposition may be related to the sense of "being watched", too. Some CPR implementations in prehospital emergency medicine occur in public where the press members are likely to take images. CPR performed in such areas may also be referred to as Out-of-Home CPR. Of course, taking control of the environment for CPR is highly different from the hospital environment in Out-of-Home CPR. At this point, according to the PECs' perceptions, the efforts of the press members for taking images adversely affect the CPR performances of healthcare providers. Presence of the press members may provoke anxiety in PECs. It is the highest rate of the study that PECs do not want any press around when performing CPR on a patient. It may be due to both anxiety of "being watched" and legal concerns including patient privacy.

In some studies, it is stated that there are differences between attitudes of health professionals towards FPDR among the various groups of health care providers (Helmer et al. 2000) FPDR is voiced more often by nurses than the physicians (Boudreaux, Francis, and Loyacano 2002; Duran et al. 2007; Madden and Condon 2007; Meyers et al. 2000; De Robertis et al. 2017). Surprisingly, the present study does not reveal such a difference. The reason why there is no such a difference among prehospital caregivers may be due to the team-based structure of prehospital emergency settings. Like many interventions in prehospital emergency settings, CPR attempt is also performed as a team, not an individual practice. This specific dynamic of prehospital settings may be the reason why there is no significant difference between the subgroups.

Ambulance personnel in Turkey should hold one of three professionals: physicians, paramedics, basic emergency medical technicians with vocational education, or/and drivers mostly focus on prehospital care. The ambulance team, consisting of these three caregivers, intervenes in all emergency cases together. The literature has shown that medical staff, especially if they are junior, have negative attitudes toward FPDR compared to other health professionals (Chapman et al. 2012). In other words, it has been stated in some studies that as the age of the profession increases, the approach to FPDR is getting more positive (Boudreaux et al. 2002; Bray et al. 2016; Chapman et al. 2012, 2013; Güneş and Zaybak 2009; Köberich et al. 2010; Ramos et al. 2016; Twibell et al. 2008). The positive perception may be related to their experience in this field. However, there is no significance in this study between clinically experienced participants and less experienced colleagues. The sample here is relatively young in their professionalism; the periods of the experience are mostly 1-3 years in participants. 
Therefore, the results are seen to be consistent with the literature. Of course, education is surely an important key to attitude change. When CPR providers are presented with FPDR education, their opinion-based beliefs may be modified to lower their guard against the issue and to improve overall support of FPDR (Abelsson and Lindwall 2018; Feagan and Fisher 2011). Therefore, education and professional experience can be defined as the factors that could change the PECs' attitudes toward FPDR in this regard.

According to the findings, there seems to be an uncertainty in participants' responses about two issues; first one, some family members' desires to be present with their loved one at the last moment. It is just about humanity, and it clearly seems very humane. Undoubtedly, PECs are also human beings, and they seem to respond to this expression in ambivalence between their professionalism and humanity aspects. They may want to use their professional knowledge and skills for their loved ones. This complex situation they experienced may have emerged as indecision in their answers in the study. The second issue is about the social and legal concerns of PECs. Allowing the family members to see or check that everything possible has been done for their loved one seems controversial for PECs. The prejudice towards patient relatives is a condition that prevents effective communication. PECs seem indecisive on the attitude they will take against people looking for deficiencies and inaccuracies in their transactions. CPR, when not properly initiated, could have negative consequences on both PECs and significant others (Chapman et al. 2013).

However, the study found significant differences in two phrases according to the gender. Women seem to be a more favourable presence with their loved one's CPR than men. It may be one perception of women can meet the needs of PECs in that critical time by participating in the CPR attempt. This may also be the result of different coping skills between men and women in highly emotional processes. The second phrase includes a difference between the genders is the intention of relatives to learn if everything is being done to their loved ones. Women are less favorable to this approach. As an implicit intention, it may be considered that relatives have a desire to take control or observe the CPR process. Somehow, women seem to be less likely to accept this idea. Alternatively, considering these issues in terms of feminist bioethics may lead to making more descriptive inferences.

In light of these results, PECs are opposed to the presence of both significant others and the press during prehospital CPR. As they think their CPR performance is affected negatively, it is necessary to decrease such additional stress on health care workers. In today's world, everyone is like a member of the press due to the widespread of social media. The probability of broadcasting in media of possible negative images creates extra stress for health workers on the same grounds. Consequently, this pressure makes a great effect on PECs during prehospital CPR. Additionally, "journalism concerns" should not interfere with the efforts of PECs "to provide qualified emergency health care". Press organizations also have important duties in this regard. It is only possible to remove some of these obstacles in order to provide effective emergency health care with the ethical sensitivity of professionals in all areas. Therefore, it is necessary to provide effective cooperation between health and the press organizations and raise some sensitivity in this interaction.

Of course, prehospital FPDR can also be affected by the following structural situations which vary from country to country; the structure of the ambulance system across the country, the practice and legislation, the culture and cultural/individual differences, and the individual sensitivity and educational issues. Of all, in some cases in prehospital settings, family presence could facilitate better understanding among relatives. In the context of the study, PECs' perceptions may be influenced by the lack of effective FPDR policy and guidelines in Turkey. Some additional studies need to be done, and further studies using larger samples in prehospital health care workers could find out more information on FPDR. 


\section{STUDY LIMITATIONS}

The study has several limitations. Firstly, the findings are based on a small sample of PECs; however, focused on an exact theme and an adequate number of the phrases are likely to be sufficient to present the attitudes. This however does not in any way narrow assumptions that may be made from study data. Secondly, it is unable to measure exactly the attitudes of health care workers directly on a controversial issue relating to ethics, law, religion, and culture. As a result, study analysis was limited to descriptive analysis. Thirdly, collecting data through a questionnaire may have led participants to be unable to express themselves efficiently. The other limitation is the data collection which occurred five years ago, and arising the question that these data are still representative. It is, of course, possible to say that attitudes could change during this period; however, this does not prevent commenting and thinking on family presence during CPR. Finally, since there are few studies in this unique setting, the questionnaire used in this study may have some parts to be improved.

\section{CONCLUSION}

It is shown in this study that prehospital emergency caregivers mostly have negative views on family presence during CPR. The study reports that emergency health care professionals mostly think "being watch" adversely affects the CPR performance among prehospital emergency caregivers. The disturbing effect on caregivers is not only related to the presence of family members or significant others but also the press. In particular, the presence of the press during CPR is considered to be an external stressor affecting the performance of the prehospital emergency medical team. Consequently, family presence and the press effect may provoke performance anxiety in prehospital team members. Prehospital emergency settings are unique, and the study shows the importance of ethics and sensitivity among prehospital caregivers who face making ethical decisions in critical situations. Family presence and the audience effect on prehospital cardiopulmonary resuscitation are crucial issues that need more attention. Further researches are needed on family presence during cardiopulmonary resuscitation in prehospital settings, especially on the presence of the press and "being watched" during CPR. It is suggested that more policy, support, and educational intervention, including ethics, are required to provide prehospital caregivers with improved guidance of family presence during resuscitation. It is also necessary to provide effective cooperation between organizations of health and the press to raise sensitivity in this interaction.

\section{ACKNOWLEDGEMENTS}

The authors thank all prehospital emergency staff for participating in the study, and Prof. İsmet Doğan for critical review of the statistics.

\section{FUNDING}

The authors received no financial support for the research. 


\section{CONFLICT OF INTEREST}

The authors declared no potential conflicts of interest with respect to the research, authorship, and/or publication of this article.

\section{REFERENCES}

Abelsson, A., and L. Lindwall. 2018. "Ethical Dilemmas in Prehospital Emergency Care from the Perspective of Specialist Ambulance Nurse Students." International Journal of Ethics Education 3(2):181-92.

Ågård, A., J. Herlitz, M. Castrén, L. Jonsson, and L. Sandman. 2012. "Guidance for Ambulance Personnel on Decisions and Situations Related to Out-of-Hospital CPR." Resuscitation 83(1):27-31.

Albarran, J., P. Moule, J. Benger, K. McMahon-Parkes, and L. Lockyer. 2009. "Family Witnessed Resuscitation: The Views and Preferences of Recently Resuscitated Hospital Inpatients, Compared to Matched Controls without the Experience of Resuscitation Survival." Resuscitation 80(9):1070-73.

American Heart Association. 2000. "Part 2: Ethical Aspects of CPR and ECC. "Circulation 102.

American Heart Association. 2015. 2015 American Heart Association Guidelines Update for Cardiopulmonary Resuscitation and Emergency Cardiovascular Care.

Badir, A., and D. Sepit. 2007. "Family Presence during CPR: A Study of the Experiences and Opinions of Turkish Critical Care Nurses.” International Journal of Nursing Studies 44(1):83-92.

Bae, H., S. Lee, and H. Y. Jang. 2008. "The Ethical Attitude of Emergency Physicians Toward Resuscitation in Korea." Journal of Emergency Medicine 34(4):485-90.

Barreto, M. D. S., H. E. Peruzzo, C. Garcia-Vivar, and S. S. Marcon. 2019. "Family Presence during Cardiopulmonary Resuscitation and Invasive Procedures: A Meta-Synthesis." Revista Da Escola de Enfermagem 53:1-13.

Berdowski, J, R. A. Berg, J. G. Tijssen, and R. W. Koster. 2010. "Global Incidences of Outof-Hospital Cardiac Arrest and Survival Rates: Systematic Review of 67 Prospective Studies." Resuscitation. 81(11):1479-1487.

Blank, R. H. 2011. "End-of-Life Decision Making Across Cultures." The Journal of Law, Medicine \& Ethics 39(2):201-14.

Boudreaux, E. D., J. L. Francis, and T. Loyacano. 2002. "Family Presence during Invasive Procedures and Resuscitations in the Emergency Department: A Critical Review and Suggestions for Future Research.” Annals of Emergency Medicine 40(2):193-205.

Bray, I., G. Kenny, D. Pontin, R. Williams, and J. Albarran. 2016. "Family Presence during Resuscitation: Validation of the Risk-Benefit and Self-Confidence Scales for Student Nurses. "Journal of Research in Nursing 21(4):306-22.

Brenner, J. M., A. L. Aswegan, L. E. Vearrier, J. B. Basford, and K. V. Iserson. 2018. "The Ethics of Real-Time EMS Direction: Suggested Curricular Content." Prehospital and Disaster Medicine 33(2):201-12. 
Brown, N. 2016. "Pre-Hospital Resuscitation: What Shall We Tell the Family?" Journal of Paramedic Practice 8(2):86-89.

Chapman, R., R. Watkins, A. Bushby, and S. Combs. 2012. "Family-Witnessed Resuscitation: Perceptions of Nurses and Doctors Working in an Australian Emergency Department." ISRN Emergency Medicine 2012:1-10.

Chapman, R., R. Watkins, A. Bushby, and S. Combs. 2013. "Assessing Health Professionals' Perceptions of Family Presence during Resuscitation: A Replication Study." International Emergency Nursing 21(1):17-25.

Demir, F. 2008. "Presence of Patients' Families during Cardiopulmonary Resuscitation: Physicians' and Nurses' Opinions." Journal of Advanced Nursing 63(4):409-16.

Dewitt, S. 2015. "Should Family-Witnessed Resuscitation Become Our Standard?” Journal of Emergency Medicine 49(4):500-502.

Duran, C. R., K. S. Oman, J. J. Abel, V. M. Koziel, and D. Szymanski. 2007. "Attitudes toward and Beliefs about Family Presence: A Survey of Healthcare Providers, Patients' Families, and Patients." American Journal of Critical Care 16(3):270-80.

ENA Clinical Practice Guideline Committee. 2012. Clinical Practice Guideline: Family Presence during Invasive Procedures and Resuscitation (Revised: 2017).

Erbay, H. 2014. "Some Ethical Issues in Prehospital Emergency Medicine." Turkish journal of emergency medicine 14(4):193-98.

Feagan, L. M. and N. J. Fisher. 2011. "The Impact of Education on Provider Attitudes Toward Family-Witnessed Resuscitation.” Journal of Emergency Nursing 37(3):231-39.

Goldberger, Z. D., B. K. Nallamothu, G. Nichol, P. S. Chan, J. R. Curtis, and C. R. Cooke. 2015. "Policies Allowing Family Presence during Resuscitation and Patterns of Care during In-Hospital Cardiac Arrest." Circulation: Cardiovascular Quality and Outcomes 8(3):226-34.

Güneş, Ü. Y., and A. Zaybak. 2009. “A Study of Turkish Critical Care Nurses' Perspectives Regarding Family-Witnessed Resuscitation.” Journal of Clinical Nursing 18(20):290715.

Helmer, S. D., R. S. Smith, J. M. Dort, W. M. Shapiro, and B. S. Katan. 2000. "Family Presence during Trauma Resuscitation: A Survey of AAST and ENA Members." Journal of Trauma - Injury, Infection and Critical Care 48(6):1015-24.

Hostler, D., S. Everson-Stewart, T. D. Rea, I. G. Stiell, C. W. Callaway, P. J. Kudenchuk, G. K. Sears, S. S. Emerson, and G. Nichol. 2011. "Effect of Real-Time Feedback during Cardiopulmonary Resuscitation Outside Hospital: Prospective, Cluster-Randomised Trial." Bmj 342(7793):371.

Jabre, P., V. Belpomme, E. Azoulay, L. Jacob, L. Bertrand, F. Lapostolle, K. Tazarourte, G. Bouilleau, V. Pinaud, C. Broche, D. Normand, T. Baubet, A. Richard-Hibon, J. Istria, A. Beltramini, A. Alheritiere, N. Assez, L. Nace, B. Vivien, L. Turi, S. Launay, M. Desmaizieres, S. W. Borron, E. Vicaut, and F. Adnet. 2013. "Family Presence During Cardiopulmonary Resuscitation.” The New England Journal of Medicine 368(11):100818.

Karlsson, M., N. Karlsson, and Y. Hilli. 2019. "Ethical Dilemmas during Cardiac Arrest Incidents in the Patient's Home." Nursing Ethics 26(2):625-37. 
Kirchhoff, C., J. Stegmaier, S. Buhmann, B. A. Leidel, P. Biberthaler, W. Mutschler, and K. G. Kanz. 2007. "Trauma Surgeons' Attitude towards Family Presence during Trauma Resuscitation: A Nationwide Survey." Resuscitation 75(2):267-75.

Köberich, S., A. Kaltwasser, O. Rothaug, and J. Albarran. 2010. "Family Witnessed Resuscitation - Experience and Attitudes of German Intensive Care Nurses." Nursing in Critical Care 15(5):241-50.

Lederman, Z. and O. Wacht. 2014. "Family Presence during Resuscitation: Attitudes of YaleNew Haven Hospital Staff." Yale Journal of Biology and Medicine 87(1).

Leske, J. S., N. S. McAndrew, and K. J. Brasel. 2013. "Experiences of Families When Present During Resuscitation in the Emergency Department After Trauma." Journal of Trauma Nursing 20(2):77-85.

Lippert, F. K., V. Raffay, M. Georgiou, P. A. Steen, and L. Bossaert. 2010. "European Resuscitation Council Guidelines for Resuscitation 2010 Section 10. The Ethics of Resuscitation and End-of-Life Decisions." Resuscitation 81(10):1445-51.

Lundsgaard, R. S. and K. S. Lundsgaard. 2018. "Cardiac Arrest Teams Perspectives on Communication and Ethical Conflicts Related to Awareness during CPR, a Focus Group Study Protocol." Scandinavian Journal of Trauma, Resuscitation and Emergency Medicine 26(1):5-7.

MacLean, S. L., C. E. Guzzetta, C. White, D. Fontaine, D. J. Eichhorn, T. A. Meyers, and P. Désy. 2003. "Family Presence during Cardiopulmonary Resuscitation and Invasive Procedures: Practices of Critical Care and Emergency Nurses." American Journal of Critical Care 12(3):246-57.

Madden, E. and C. Condon. 2007. "Emergency Nurses' Current Practices and Understanding of Family Presence During CPR.” Journal of Emergency Nursing 33(5):433-40.

Masa'Deh, R., A. Saifan, S. Timmons, and S. Nairn. 2014. "Families' Stressors and Needs at Time of Cardio-Pulmonary Resuscitation: A Jordanian Perspective." Global Journal of Health Science 6(2):72-85.

McClement, S. E., W. M. Fallis, and A. Pereira. 2009. "Family Presence during Resuscitation: Canadian Critical Care Nurses' Perspectives." Journal of Nursing Scholarship 41(3):233-40.

McLaughlin, K., V. Melby, and V. Coates. 2013. "Family-Centred Care during Resuscitation Events." Emergency Nurse 21(3):28-34.

Meyers, T. A., B. J. Eichhorn, C. E. Guzzetta, A. P. Clark, J. D. Klein, E. Taliaferro, and A. Calvin. 2000. "Family Presence during Invasive Procedures and Resuscitation: The Experience of Family Members, Nurses, and Physicians." American Journal of Nursing 100(2):32-42.

Mian, P., S. Warchal, S. Whitney, J. Fitzmaurice, and D. Tancredi. 2007. "Impact of a Multifaceted Intervention on Nurses' and Physicians' Attitudes and Behaviors Toward Family Presence During Resuscitation." Critical Care Nurse 27(3):52-61.

Moreland, P. 2005. "Family Presence during Invasive Procedures and Resuscitation in the Emergency Department: A Review of the Literature." Journal of Emergency Nursing 31(1):58-72. 
Al Mutair, A., V. Plummer, A. P. O’Brien, and R. Clerehan. 2014. "Attitudes of Healthcare Providers towards Family Involvement and Presence in Adult Critical Care Units in Saudi Arabia: A Quantitative Study." Journal of Clinical Nursing 23(5-6):744-55.

Pankop, R., K. Chang, J. Thorlton, and T. Spitzer. 2013. "Implemented Family Presence Protocols: An Integrative Review." Journal of Nursing Care Quality 28(3):281-88.

Parial, L. L. B., G. C. S. Torres, and J. R. B. Macindo. 2016. "Family Presence During Resuscitation Benefits-Risks Scale (FPDR-BRS): Instrument Development and Psychometric Validation.” Journal of Emergency Nursing 42(3):213-23.

Ramos, M., J. Z. Diaz, G. G. De Jesús, D. Moulia, and T. Quest. 2016. "Puerto Rican Emergency Department Clinicians' Knowledge, Practice and Attitudes Regarding Family Witness Resuscitation (SA527A)." Journal of Pain and Symptom Management, 51(2): 398.

De Robertis, E., G. M. Romano, J. Hinkelbein, O. Piazza, and G. Sorriento. 2017. "Family Presence during Resuscitation: A Concise Narrative Review." Trends in Anaesthesia and Critical Care 15(2017):12-16.

Sak-Dankosky, N., P. Andruszkiewicz, P. R. Sherwood, and T. Kvist. 2015. "Factors Associated with Experiences and Attitudes of Healthcare Professionals towards FamilyWitnessed Resuscitation: A Cross-Sectional Study." Journal of Advanced Nursing 71(11):2595-2608.

Sasson, C., M. A. Rogers, J. Dahl, and A. L. Kellermann. 2010. "Predictors of Survival from Out-of-Hospital Cardiac Arrest a Systematic Review and Meta-Analysis." Circulation: Cardiovascular Quality and Outcomes 3(1):63-81.

De Stefano, C., D. Normand, P. Jabre, E. Azoulay, N. Kentish-Barnes, F. Lapostolle, T. Baubet, P. G. Reuter, N. Javaud, S. W. Borron, E. Vicaut, and F. Adnet. 2016. "Family Presence during Resuscitation: A Qualitative Analysis from a National Multicenter Randomized Clinical Trial." PLoS ONE 11(6):1-12.

Strasen, J., S. L. Van Sell, and S. Sheriff. 2016. "Family Presence during Resuscitation." Nursing Critical Care 11(4):42-46.

Tennyson, C. D. 2019. "Family Presence during Resuscitation: Updated Review and Clinical Pearls." Geriatric Nursing 40(6):645-47.

Torabi, M., F. Borhani, A. Abbaszadeh, and F. Atashzadeh-Shoorideh. 2019. "Ethical Decision-Making Based on Field Assessment: The Experiences of Prehospital Personnel." Nursing Ethics 26(4):1075-86.

Twibell, R. S., D. Siela, C. Riwitis, J. Wheatley, T. Riegle, D. Bousman, S. Cable, P. Caudill, S. Harrigan, R. Hollars, D. Johnson, and A. Neal. 2008. "Nurses' Perceptions of Their Self-Confidence and the Benefits and Risks of Family Presence during Resuscitation." American Journal of Critical Care : An Official Publication, American Association of Critical-Care Nurses 17(2):101-11; quiz 112.

Walker, W. M. 2014. "Emergency Care Staff Experiences of Lay Presence during Adult Cardiopulmonary Resuscitation: A Phenomenological Study." Emergency Medicine Journal : EMJ 31(6):453-58.

Wik, L., J. Kramer-Johansen, H. Myklebust, H. Sørebø, L. Svensson, B. Fellows, and P. A. Steen. 2005. "Quality of Cardiopulmonary Resuscitation During Out-of-Hospital Cardiac Arrest." JAMA 293(3):299. 
Yanturali, S., G. Ersoy, A. Yuruktumen, E. Aksay, S. Suner, Y. Sonmez, D. Oray, N. Colak, and A. H. Cimrin. 2005. "A National Survey of Turkish Emergency Physicians Perspectives Regarding Family Witnessed Cardiopulmonary Resuscitation." International Journal of Clinical Practice 59(4):441-46.

Zavotsky, K. E., J. McCoy, G. Bell, K. Haussman, J. Joiner, K. K. Marcoux, K. Magarelli, K. Mahoney, L. Maldonado, K. A. Mastro, A. Milloria, L. M. Tamburri, and D. Tortajada. 2014. "Resuscitation Team Perceptions of Family Presence During CPR." Advanced Emergency Nursing Journal 36(4):325-34.

\section{HASAN ERBAY}

Department of History of Medicine and Ethics

Faculty of Medicine

Afyonkarahisar Health Sciences University, Turkey

*Corresponding author: hasanerbay@yahoo.com 